\title{
Reconocimiento de expresión facial emocional en el trastorno de déficit de atención e hiperactividad en la infancia
}

\author{
Mireia Oliva-Macías ${ }^{1}$, Pamela Parada-Fernández ${ }^{1}$, Imanol Amayra², \\ Esther Lázaro² y Juan F. López-Paz \\ ${ }^{1}$ Universidad Europea del Atlántico, Santander, España \\ ${ }^{2}$ Universidad de Deusto, Bilbao, España
}

Recognition of emotional facial expression in attention deficit/hyperactivity disorder in childhood

\begin{abstract}
The main symptoms of attention deficit/hyperactivity disorder (ADHD) are inattention, hyperactivity and impulsivity. In addition to cognitive and behavioral deficits present in ADHD, having difficulties in social skills has also been observed in different studies. The objective of this study was to analyze performance in recognizing emotional facial expression in this group. For this, a clinical group with ADHD was compared to a control group. Emotional facial expression recognition tools were applied. No statistically significant differences were found between groups in non-contextualized static emotions. However, differences were found in non-contextualized dynamic emotions, contextualized scenarios and secondary social skills. In addition, a more comprehensive analysis identified a subgroup of children with ADHD that performed better than the other ADHD group of children and similarly to the control group.
\end{abstract}

Keywords: Social skills; virtual reality; recognition of emotional facial expression; theory of mind; attention deficit disorder and hyperactivity.

Resumen: Los síntomas principales del trastorno de déficit de atención/hiperactividad (TDAH) son la inatención, la hiperactividad y la impulsividad. Además de los déficits cognitivos y conductuales presentes en el TDAH, se ha observado en diferentes estudios la presencia de dificultades en las habilidades sociales. El objetivo de este estudio fue analizar el rendimiento en el reconocimiento de expresión facial emocional en este colectivo. Para ello, se comparó un grupo clínico con TDAH con un grupo control. Se emplearon herramientas de reconocimiento de expresión facial emocional. No se encontraron diferencias estadísticamente significativas en las emociones estáticas no contextualizadas entre los dos grupos. Sin embargo, sí se encontraron diferencias en las emociones dinámicas no contextualizadas, en escenarios contextualizados y habilidades sociales secundarias. Además, un análisis más exhaustivo identificó un subgrupo de niños con TDAH con un rendimiento similar al presentado por el grupo control y superior al resto de niños del grupo TDAH.

Palabras clave: Habilidades sociales; realidad virtual; reconocimiento de expresión facial emocional; teoría de la mente; trastorno de déficit de atención e hiperactividad.

Recibido: 30 de enero 2018; aceptado: 09 de mayo 2018

Correspondencia: Mireia Oliva Macías, Facultad de Ciencias de la Salud (Grado en Psicología), Universidad Europea del Atlántico, Isabel Torres 21,39011 Santander, España. Correo-e: mireia.oliva@uneatlantico.es Agradecimientos: A las familias de los niños con TDAH que se han puesto a nuestra disposición para poder realizar este estudio. Por su continuo esfuerzo por mejorar su calidad de vida y poner en disposición del mundo de la investigación cualquier aspecto que este en sus manos para avanzar y ayudar a futuras familias en la evaluación precoz y mejora de los tratamientos.

\section{Introducción}

El trastorno de déficit de atención e hiperactividad (TDAH) es un trastorno del neurodesarrollo diagnosticado principalmente en población infantojuvenil. Se caracteriza por síntomas como el déficit de atención, hiperactividad e impulsividad. Teniendo en cuenta estos criterios, y tomando como referencia el periodo de los últimos seis meses, según el DSM-IV-TR y el DSM-5 es 
posible identificar tres subtipos (en el DSM-IV-TR) o especificadores (en el DSM-5) de TDAH: tipo combinado, tipo con predominio del déficit de atención y tipo con predominio hiperactivo-impulsivo (American Psychiatric Association, 1994; 2014). Su prevalencia en población normal a nivel mundial oscila entre un $2 \%$ y un $18 \%$, aunque ésta varía en función de la población estudiada y del sistema de evaluación. En niños ${ }^{1}$ en edad escolar se estima entre el 3-7\% (Polanczyk, Lima, Horta, Biederman, y Rohde, 2007), siendo mucho más frecuente en los varones que en las mujeres, en una proporción de 4:1 (Rodríguez, 2010; Rubiales, Urquijo, Said, y Macbeth, 2017).

Junto con los tres síntomas principales mencionados, es importante destacar diferentes aspectos que se ven afectados en la vida de estos niños. Uno de ellos es la dificultad para establecer relaciones con sus iguales y con adultos (Nixon, 2001). Este hecho les lleva a ser impopulares entre sus compañeros, lo que acaba produciendo baja autoestima y una percepción de baja autoeficacia en sus acciones (Garza, Núñez, y Vladimirsky, 2007; Villanueva-Bonilla y Ríos-Gallardo, 2018). Además, son frecuentes los trastornos emocionales como la depresión, el trastorno bipolar y la distimia y cerca de un $20 \%$ presenta dificultades específicas de aprendizaje (Brown, 2009).

Así mismo, dentro de la sintomatología del TDAH, se ha dado mayor importancia a los aspectos relacionados con el rendimiento escolar, es decir, a los aspectos cognitivos, que a las alteraciones emocionales, las cuales han estado en un segundo lugar, siendo limitado su estudio y quedando muchas cuestiones por resolver en relación a las dificultades emocionales en el TDAH.

Uno de los aspectos que se ha planteado desde la investigación y la clínica es a qué se deben las dificultades de estos niños para relacionarse con sus iguales. Es cierto que la impulsividad puede entorpecer las habilidades sociales, pero, más allá de eso, algunos estudios sugieren que los niños con TDAH tienen importantes dificultades para reconocer y comprender la información afectiva (Sinzig, Morsch, y Lehmkuhl, 2008).

El reconocimiento de las expresiones faciales emocionales es un componente clave de la cognición social (Williams, Hermes, Palmer, Kohn, Clarke, Keage, y Gordon, 2008). El reconocimiento preciso de las expresiones faciales ayuda a la comprensión de los sentimientos e intenciones de los otros y a dar una respuesta adecuada ante las situaciones sociales. De hecho, la capacidad para

\footnotetext{
${ }^{1}$ A lo largo del artículo, salvo que se indique lo contrario, se utiliza el genérico «niños» para referirse tanto a niños como a niñas.
}

interpretar y responder de forma apropiada a las emociones de los otros es crucial para establecer relaciones interpersonales adaptadas (Fairchild, Van Goozen, Calder, Stollery, y Goodyer, 2009; Nakhuntina, Borod, y Zgaljardic, 2006).

Es por ello que, en relación al reconocimiento emocional en personas con diagnóstico de TDAH, las principales teorías sostienen que el reconocimiento emocional no debe estar afectado. Sin embargo, ciertos síntomas característicos del trastorno, como la inatención y la impulsividad, pueden dificultar el reconocimiento de distintos estímulos emocionales como es el caso de las expresiones faciales y la prosodia (pronunciación y entonación de las palabras) (Albert, López-Martín, Fernández-Jaén, y Carretil, 2008). Diversos estudios concluyen que los niños con TDAH son menos exactos reconociendo emociones, especialmente emociones desagradables como el miedo, el enfado y la tristeza (Pelc, Kornreich, Foisy, y Dan, 2006). Otra de las variables a tener en cuenta en el reconocimiento de la expresión facial emocional del colectivo que presenta TDAH es el tiempo de reacción. Existen escasos estudios que hayan medido esta variable. En un primer momento se defendía la hipótesis de que las personas diagnosticadas con TDAH tendrían una mayor rapidez a la hora de responder a los estímulos de reconocimiento facial emociona. Sin embargo, se ha encontrado una mayor demora de esta población en responder a este tipo de estímulos (Kats-Gold, Besser, y Priel, 2007; Rapport, Friedman, Tzelepis, y Van Voorhis, 2002).

En relación a la evaluación del reconocimiento de expresiones faciales emocionales, la realidad virtual abre un nuevo camino evaluando en un entorno ecológico y dinámico, ya que simula entornos reales donde los sujetos pueden interaccionar. La realidad virtual es entendida como el espacio digital que sustituye al entorno físico y a las experiencias sensoriales (Fox, Arena, y Bailenson, 2009). A nivel metodológico, la realidad virtual permite controlar experimentalmente estímulos como, por ejemplo, la duración de los estímulos presentados, el orden de presentación, el tipo de estimulación, las alternativas de respuesta, los entornos, los personajes, las instrucciones y la retroalimentación. Además, facilita la recogida automatizada de resultados como el tipo de respuesta, los tiempos de reacción, la dirección de la mirada o la repetición de la instrucción.

En el ámbito del TDAH la realidad virtual está siendo utilizada en diversos ámbitos como el de la evaluación (Gutiérrez-Maldonado, Letosa-Porta, Rus-Calafell, y Peñaloza-Salazar, 2009), la rehabilitación de la atención (Anton, Opris, Dobrean, David, y Rizzo, 2009) o las interacciones y relaciones sociales (Fenstermacher, Olym- 
pia, y Sheridan, 2006; Gutierrez-Maldonado, Alsina-Jurnet, Carvallo-Becíu, Letosa-Porta, y Magallón-Neri, 2007). Por ejemplo, las tareas de atención sostenida tradicionales son útiles para detectar TDAH; sin embargo, a pesar de que muestran una buena validez interna, su validez ecológica es limitada. Por ello, su adaptación al realismo de la realidad virtual mejora la calidad del instrumento (Adams, Flannery, Finn, Rizzo, y Moes, 2009). Por ejemplo, Gutiérrez-Maldonado et al., (2009) hace uso de esta validez ecológica para crear una clase de realidad virtual con la intención de evaluar aspectos del TDAH en la vida real. Otro de los ámbitos de aplicación la realidad virtual ha sido el del entrenamiento en habilidades sociales. El entrenamiento en habilidades sociales puede hacerse mediante dinámicas grupales pero estas intervenciones pueden tener consecuencias negativas, como cambios en las actitudes sobre la conducta antisocial, la identificación con iguales que muestran conductas de riesgo, etc. La realidad virtual, sin embargo, brinda la oportunidad de controlar estos aspectos y prevenir consecuencias no deseadas (Fenstermacher et al., 2006).

Teniendo en cuenta la limitación de investigaciones que han estudiado las habilidades sociales en niños diagnosticados con TDAH, este estudio pretende comparar la capacidad de reconocer expresiones faciales emocionales y el tiempo de reacción asociado a dicho reconocimiento entre niños con TDAH y niños sin TDAH mediante una prueba ecológica de realidad virtual.

\section{Método}

\section{Participantes}

La muestra del presente estudio estuvo compuesta por 236 niños divididos en dos grupos: grupo clínico y grupo control. El grupo clínico estuvo formado por 118 niños diagnosticados de TDAH con edades comprendidas entre los 7 y los 11 años. La edad media fue de 9.51 $(D T=1.2)$. De forma congruente con los estudios de prevalencia, la muestra estuvo compuesta por un mayor porcentaje de niños $(84.7 \% ; n=100)$ que de niñas $(15.3 \% ; n=18)$ diagnosticados de TDAH. Además, en la muestra imperaba el subtipo combinado $(73.7 \% ; n=87)$ frente al desatento $(20.3 \% ; n=24)$ o al hiperactivo-impulsivo $(5.9 \% ; \mathrm{n}=7)$.

Los participantes fueron derivados de diferentes entidades, entre ellas asociaciones de afectados, centros de salud mental infanto-juveniles y departamentos psicopedagógicos escolares de diferentes provincias a nivel de España. Respecto al tratamiento farmacológico que los participantes estaban siguiendo en el momento de la evaluación, el $77.1 \%$ recibían metilfenidato, el $4.2 \%$ medi- cina alternativa (p.ej., homeopatía), el 2.5\% atomoxetina, el $0.8 \%$ antidepresivos tricíclicos y el $15.3 \%$ no tomaba medicación.

La muestra clínica fue seleccionada atendiendo a los siguientes criterios de inclusión: a) los síntomas debían manifestarse en diferentes ambientes; b) los síntomas debían estar presentes antes de los 7 años; c) los síntomas no podían ser explicados por otras causas (p.ej., problemas emocionales o baja capacidad); d) los síntomas producían desadaptación en el ambiente; e) los criterios anteriores se describían en sus informes correspondientes (realizados por los neurólogos del sistema sanitario público); f) el niño estaba tomando su correspondiente tratamiento en el momento de la evaluación; g) el niño estaba escolarizado; h) el niño cursaba estudios de $3^{\circ}, 4^{\circ}, 5^{\circ}$ o $6^{\circ}$ de educación primaria obligatoria y i) el niño estaba familiarizado con el entorno informático (realizaba manejo de las herramientas informáticas en el colegio).

El grupo control se obtuvo de una muestra mayor de 1700 niños en los que se habían validado los instrumentos FEEL y Neuro-e-Motion (ver apartado de instrumentos para más información sobre los mismos). De esta muestra de 1.700 niños se seleccionó una muestra lo más parecido posible a la del grupo clínico. De esta forma, se seleccionaron 118 niños no diagnosticados de TDAH con edades entre 7 y 11 años, con una edad media de 9.5 y una desviación típica de 1.2. El porcentaje de niños fue de $84.7 \%(n=100)$ y el de niñas de $15.3 \%(n=18)$. Los criterios de inclusión correspondientes a esta muestra fueron los siguientes: a) escolarización obligatoria; b) cursar estudios de $3^{\circ}, 4^{\circ}, 5^{\circ}$ o $6^{\circ}$ de educación primaria obligatoria; c) estar familiarizado con el entorno informático (realizaba manejo de las herramientas informáticas en el colegio).

Los participantes de ambos grupos no se diferenciaron significativamente en ninguna de las variables de apareamiento o agrupación, edad y género.

\section{Procedimiento}

Se realizaron contactos con asociaciones de familiares de niños con TDAH y departamentos de orientación/ psicología de colegios. El $60 \%$ de la muestra fue evaluada a través de asociaciones de familiares y el $40 \%$ a través de colegios concertados y privados.

En el caso del grupo clínico, se contactaba por teléfono con el responsable del departamento de psicología de la asociación de familiares y, a demanda, se les facilitaba la información vía correo electrónico para que pudieran explicarla en el centro. Posteriormente, y tras recibir la aceptación de la asociación de participar en el estudio, el 
equipo que realizaba la evaluación se trasladaba a la asociación para realizar una presentación a los profesionales y padres sobre el proyecto y se les explicaba el procedimiento a llevar a cabo. En esta reunión se les daba una carta explicativa a las familias y el consentimiento informado. Las familias interesadas contactaban con los evaluadores y una vez firmado el consentimiento informado por parte de los padres se fijaban las fechas de evaluación. El día de la evaluación se realizaba a los padres una breve entrevista para la recogida de datos sociodemográficos y posteriormente se pasaba a evaluar a los niños con la prueba FEEL y Neuro-e-Motion, siguiendo ese orden.

En el caso de realizar el contacto con los colegios, el procedimiento a seguir era similar al realizado con las asociaciones: se contactaba con el psicólogo responsable del departamento en el colegio a quien se le presentaba el proyecto con sus finalidades y el procedimiento a realizar. Una vez el colegio aceptaba la participación, el equipo se trasladaba para una presentación a los profesores interesados. Posteriormente se les facilitaba una carta informativa junto con el consentimiento informado para que facilitaran a los padres. Si las familias consentían la participación en el estudio, una vez firmada la carta de consentimiento informado y completada la hoja con los datos sociodemográficos básicos, se citaba a los niños para la evaluación con el FEEL y el Neuro-e-Motion, en ese orden.

En el caso del grupo control, era necesario un consentimiento por parte de los padres que habían recibido la información del estudio a través de los colegios. Una vez firmado el consentimiento informado se les facilitaba una breve entrevista para recoger la información sobre los datos sociodemográficos de los menores. Las pruebas fueron aplicadas de forma individual a cada niño en el mismo orden que el estipulado para los niños con TDAH.

Para la evaluación de los niños se entrenaron dos evaluadores y éstos llevaron a cabo las evaluaciones en un periodo de seis meses. La evaluación con cada niño tuvo una duración aproximada de 40 minutos.

\section{Instrumentos}

Facially Expressed Emotion Labeling (FEEL; Kessler, Bayerl, Deighton, y Traue, 2002). La prueba FEEL es una prueba computarizada que mide la capacidad de reconocer las emociones básicas (ira, asco, miedo, alegría, sorpresa y tristeza) en las expresiones faciales. Cada emoción básica es mostrada de forma estática en cuatro personas (dos mujeres y dos hombres) de raza caucásica (16 imágenes) y de origen asiático (16 imáge- nes). La prueba comienza con la presentación de una pre-serie de seis imágenes a modo de ensayo en la que cada emoción se presenta una vez. Esto ayuda a los sujetos a familiarizarse y orientarse con la prueba. Esta fase de ensayo es seguida por la prueba real que incluye 42 imágenes ( 7 imágenes de cada una de las 6 emociones). La presentación del estímulo y la escala de medida de las 42 imágenes posteriores siguen el mismo procedimiento en cuanto a empezar con una fase de ensayo. Antes de presentar la expresión facial emocional que tiene que ser identificada, se presenta una expresión facial neutra durante 1.5 segundos. La secuencia de imágenes para cada ensayo se determina mediante un generador aleatorio para evitar un efecto de la secuencia. Se evalúa la precisión de respuesta, siendo la puntuación mínima 0 y la máxima 42 y el tiempo de reacción ante el estímulo. Respecto a sus cualidades psicométricas,la prueba ha sido aplicada a más de 400 participantes, obteniendo un índice de alfa de Cronbach de $\alpha=0.77$ (Lárazo et al., 2016).

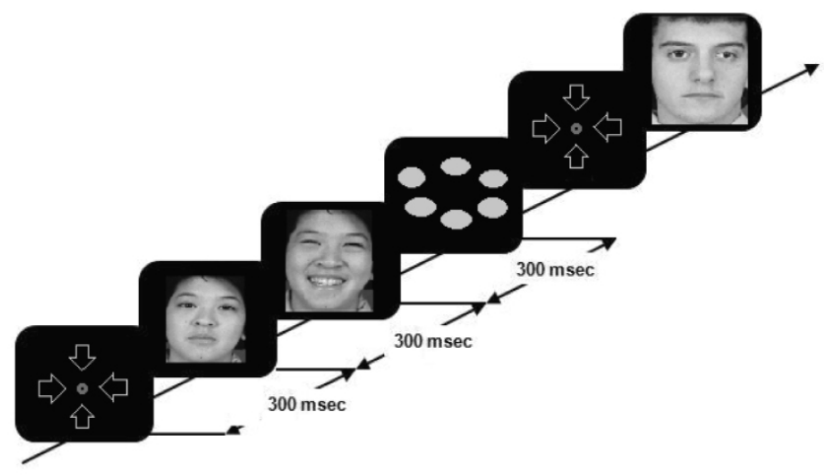

Figura 1. Ejemplo estímulos FEEL.

Deusto-e-Motion (Lázaro et al., 2016). El Deusto-e-Motion es un instrumento de realidad virtual cuyo objetivo es evaluar la capacidad empática y de reconocimiento en relación con la expresión de las emociones básicas propias y de los demás. Integra un editor consistente en una ficha de datos de cada caso en la que el evaluador tiene la posibilidad de elegir entre diferentes series de ítems relacionados con expresiones faciales estáticas, dinámicas y escenarios virtuales. Cada uno de los escenarios virtuales incluye una descripción de la situación presentada y seis opciones de respuesta (alegría, tristeza, asco, miedo, ira y sorpresa). Éstas quedan registradas de forma automática después de ser medidas mediante la siguiente escala medida: respuestas correctas, respuesta elegida por parte del sujeto y tiempo de reacción en milisegundos. El test está compuesto de varios apartados, detallados a 
continuación. En primer lugar, se realiza un test de fijación ocular hacia un punto central que va cambiando de color durante 200 milisegundos. Este test sirve para que el sujeto se familiarice con el entorno del instrumento. La siguiente tarea se divide en cuatro bloques principales en los que, en total, se presentan 14 ítems de expresiones faciales emocionales estáticas, 10 de expresiones faciales emocionales dinámicas y 20 escenarios virtuales en los que se presentan situaciones relacionadas con las habilidades sociales de la vida cotidiana y con la empatía. En cuanto a las medidas

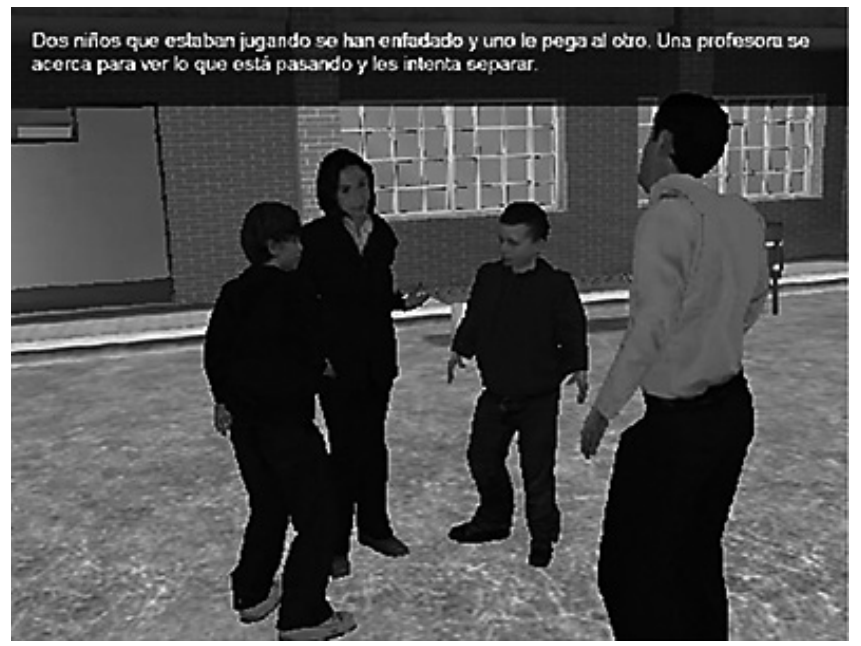

psicométricas, se encontró entre el Deusto-e-Motion caras total y FEEL caras total una validez concurrente significativa $(r=.339 ; p<.01)$; y una validez concurrente significativa entre Neuro-e-Motion tiempo de reacción total y FEEL tiempo de reacción total $(\mathrm{r}=$ $.508 ; p<.01)$. En cuanto a la fiabilidad, en el presente estudio se encontró un alpha de Cronbach de $\alpha=.85$ en la escala de reconocimiento emocional, de $\alpha=.84$ en cuanto a la escala de tiempo de reacción en el reconocimiento emocional y $\alpha=.86$ en la escala de tiempo de reacción de escenarios virtuales.

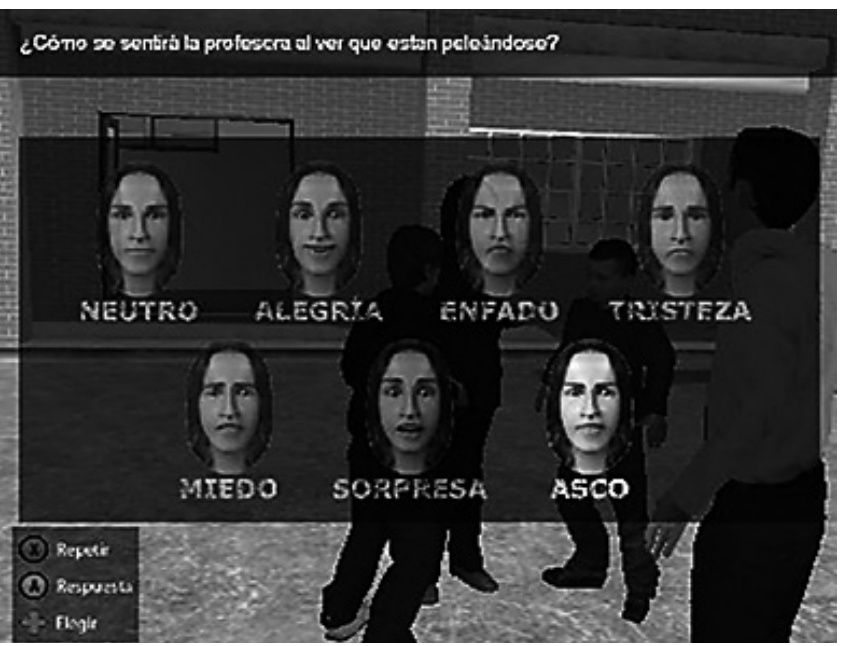

Figura 2. Ejemplos estímulos Neuro-e-Motion

\section{Análisis Estadístico}

En un primer momento se analizó la distribución normal de cada una de las variables estudiadas mediante la prueba de Kolmogorov-Smirnov y Shapiro Wilk. Aquellas variables que no siguieron una distribución normal se trataron de trasformar mediante las indicaciones de Field (2005) con el objetivo de utilizar pruebas paramétricas, no siendo efectivo. Por otro lado, con el objetivo de analizar las diferencias existentes en el conjunto de variables estudiadas entre el grupo clínico y control, se utilizó la prueba no paramétrica para dos muestras independientes U de Mann-Withney.

Para el análisis de diferencias entre los subtipos de TDAH, se utilizó la prueba no paramétrica para más de dos muestras independientes $\mathrm{H}$ de Kruskal-Wallis, que se basa en la comparación de medianas. En este sentido, cuando se hallaron diferencias estadísticamente significativas, se efectuaron comparaciones múltiples por parejas aplicando la prueba U de Mann-Whitney. Por último, se realizó un análisis de función discriminante. Este análisis identifica las características que permiten diferen- ciar a dos o más grupos y crear una función capaz de distinguir con la mayor precisión posible a los miembros de uno u otro grupo. Este análisis se realizó según diagnóstico, tipo de TDAH y edad.

Para todos los contrastes de hipótesis se aceptó un valor de significación estadística $(p)$ menor o igual a 0.05 para un nivel de confianza del $95 \%$.

\section{Resultados}

\section{Variables de reconocimiento facial emocional en escenarios no-contextualizados}

Fotografías estáticas. En la precisión de respuesta no se observaron diferencias significativas en precisión de respuesta en las diferentes emociones presentadas mediante fotografías. Sin embargo, se hallaron diferencias estadísticamente significativas entre el grupo clínico y el grupo control en el tiempo de reacción de miedo $(U=$ 5401; $z=-2.690 ; p=.007)$, en el tiempo de reacción de asco $(U=5503 ; z=-2.492 ; p=.013)$ y en el tiempo de reacción de enfado $(U=5370 ; z=-2.751 ; p=.006)$. 
Herramienta virtual. Se apreciaron diferencias estadísticamente significativas en precisión de respuesta en el total de las caras $(U=2962, z=-2.354, p=.019)$, tiempo de reacción en el total de caras $(U=2411 ; z=-4.013 ; p<$ $.001)$, precisión de respuesta en emociones dinámicas $(U=$ 5642; $z=-2.364 ; p=.018)$, tiempo de reacción en emociones dinámicas $(U=5712 ; z=-2.186 ; p=.029)$, precisión de respuesta en emociones estáticas $(U=2988 ; z=-2.285$; $p=.022)$, y tiempo de reacción en tiempo de reacción de emociones estáticas $(U=2422 ; z=-3.98 ; p<.001)$.

\section{Variables de reconocimiento facial en escenarios contextualizados}

Herramienta virtual. En la segunda parte de la herramienta computarizada no se pueden establecer respuestas correctas o incorrectas, ya que el participante debe evaluar el significado emocional expresado en forma de expresión facial de una situación dinámica expuesta a través de la realidad virtual. Sin embargo, se ha realizado un análisis de frecuencias en ambos grupos para poder observar cualitativamente la tendencia de respuesta emocional. Para observar la diferencia de medias en la precisión de respuesta, se realizó la prueba Chi cuadrado para variables cualitativas. En este caso se encontraron diferencias significativas en los siguientes ítems: $8.1\left(\mathrm{X}^{2}(6)\right.$ $=12.973 ; p=.043)$ y $24.1\left(\mathrm{X}^{2}(6)=17.054 ; p=.009\right)$. En cuanto al tiempo de reacción, se encontraron diferencias estadísticamente significativas en los siguientes ítems: $8.2(U=5682 ; z=-2.244 ; p=.025), 9.2(U=5602 ; z=$ $-2.386 ; p=.017), 10.2(U=4923 ; z=-3.710 ; p<.001)$, $15.1(U=5409 ; z=-2.771 ; p=.006), 15.2(U=5812 ; z$ $=-1.993 ; p=.046), 16(U=5745 ; z=-2.123 ; p=.034)$, $17(U=5595 ; z=-2.412 ; p=.016), 20.1(U=5351 ; z=$ $-2.883 ; p=.004), 23.1(U=5220 ; z=-3.136 ; p=.002)$, $25.2(U=5551 ; z=-2.496 ; p=.013), 26(U=5548 ; z=$ $-2.503 ; p=.012)$ y $27(U=5815 ; z=-1.987 ; p=.047)$.

\section{Comparación de las variables clínicas entre los tres subtipos clínicos}

No se encontraron diferencias estadísticamente significativas entre los tres subtipos en el reconocimiento de las diferentes emociones básicas presentadas a través de fotografías, ni en la herramienta virtual en las caras estáticas y dinámicas. Tampoco se observaron en los diferentes tiempos de reacción.

\section{Análisis función discriminante según diagnóstico}

En este análisis se estableció como variable dependiente el diagnóstico (control - TDAH) y como variables independientes las puntuaciones totales del reconocimiento de emociones básicas en formato fotografía, y total de reconocimiento emocional en la herramienta virtual. Según el análisis se observó que las 4 variables que discriminan entre estos grupos son: tiempo de respuesta del total de las caras, tiempo de respuesta en emoción de sorpresa y miedo, y por último las caras presentadas de forma estática.

Tabla 1. Resultado del análisis discriminante, mediante el método de etapas para los grupos control y clínico.

\begin{tabular}{lll}
\hline Paso & Variable & Lambda de Wilks \\
\hline 1 & Deusto-e-Motion caras total-TR & $.930^{* *}$ \\
2 & FEEL sorpresa-TR & $.903^{* *}$ \\
3 & FEEL miedo-TR & $.850^{* *}$ \\
4 & Deusto-e-Motion estáticas & $.821^{* *}$ \\
\hline Exactitud de la clasificación $(68.8 \%)$ & \\
\hline${ }^{*} p<.05, * * p<.01$. \\
\hline
\end{tabular}

\begin{tabular}{|c|c|c|}
\hline & \multicolumn{2}{|c|}{ Grupo pronosticado } \\
\hline & Clínico & Control \\
\hline \multicolumn{3}{|c|}{ Grupo actual } \\
\hline Clínico & $55(60.4 \%)$ & $36(39.6 \%)$ \\
\hline Control & $18(22 \%)$ & $64(78 \%)$ \\
\hline
\end{tabular}

La Lambda del modelo de las 4 variables fue de $\lambda=$ .821. El autovalor obtenido está bastante próximo a cero (.218) y la correlación canónica .423 es moderada, por lo que debemos suponer que las variables discriminantes utilizadas distinguen de forma moderada a los 2 grupos. Los coeficientes de función discriminante canónicos estandarizados se relacionan con una única función que engloba las cuatro variables discriminantes y que denominamos «reconocimiento de expresión facial». Hay que precisar que el componente más discriminante en estas variables es el tiempo de reacción, si bien en un segundo lugar la precisión de respuesta en caras estáticas tiene su importancia. En la función centroide en grupo se obtienen que el tiempo de reacción en el grupo clínico (.443) es mayor que el grupo control (-.486). Finalmente, en cuanto al comportamiento de los grupos, este caso el $60.4 \%$ del grupo clínico se comporta realmente como clínico mientras que el $39.6 \%$ lo hace como parte de la muestra control. En el caso de la muestra control es el $78 \%$ el que se comporta como sujetos control mientras que el $22 \%$ lo hace como clínico. En resumen, la exactitud de clasificación es de un $68.8 \%$ en el caso del grupo clínico y control. 
Una vez realizado el análisis función discriminante según diagnóstico se han diferenciado 4 grupos. En el caso del grupo diagnosticado de TDAH, el $69.49 \%$ se comporta como TDAH esperado, mientras que el $30.5 \%$ lo hace como la muestra control. En el caso del grupo control, el $85.59 \%$ se comporta como tal mientras que el $14.4 \%$ se comporta como TDAH. Es decir, se establecen 4 grupos diferenciados: TDAH puro, TDAH como control, control puro y control como TDAH. Teniendo en cuenta esto es interesante analizar las diferencias entre los grupos según el criterio psicométrico. Al comparar los cuatro grupos se observaron diferencias estadísticamente significativas en precisión de respuesta de la emoción de miedo y tristeza. Así mismo, en el tiempo de reacción se encontraron diferencias en miedo, alegría, sorpresa, asco, tristeza y enfado. En el caso de la herramienta virtual se observaron diferencias en la precisión de respuestas en el reconocimiento total, estáticas y dinámicas. En cuanto al tiempo de reacción se observaron diferencias en: caras totales, estáticas y dinámicas.

Tabla 2. Diferencias entre los cuatro grupos según criterios psicométricos.

\begin{tabular}{lcc}
\hline \multicolumn{1}{c}{ Variable } & $\chi^{2}$ & $p$ \\
\hline PR Miedo & 12.500 & $.006^{* *}$ \\
PR Tristeza & 15.700 & $.001^{* *}$ \\
TR Miedo & 30.136 & $.000^{* * *}$ \\
TR Alegría & 18.951 & $.000^{* * *}$ \\
TR Sorpresa & 11.619 & $.009^{* *}$ \\
TR Asco & 17.683 & $.001^{* *}$ \\
TR Tristeza & 20.267 & $.000^{* * *}$ \\
TR Enfado & 18.588 & $.000^{* * *}$ \\
PR Caras total & 30.921 & $.000^{* * *}$ \\
PR Caras estáticas & 28.801 & $.000^{* * *}$ \\
PR Caras dinámicas & 16.785 & $.001^{* *}$ \\
TR Caras total & 52.522 & $.000^{* * *}$ \\
TR Caras estáticas & 48.520 & $.000^{* * *}$ \\
TR Caras dinámicas & 43.441 & $.000^{* * *}$ \\
PR HHSS secundarias & 8.137 & $.043^{*}$ \\
TR HHSS secundarias & 18.099 & $.000^{* * *}$ \\
\hline
\end{tabular}

Nota. PR: precisión respuesta; TR: tiempo reacción.

${ }^{*} p<.05,{ }^{* *} p<.01, * * * p<.001$.

\section{Discusión}

El objetivo principal del presente estudio fue analizar las diferencias existentes en los niños con y sin TDAH en el rendimiento de un conjunto de pruebas que evalúan el reconocimiento de emociones faciales emocionales contextualizadas y no contextualizadas con caras estáticas y dinámicas. Para ello, se tuvo en cuenta tanto la precisión de respuesta como el tiempo de reacción.

En relación con la tasa de aciertos en el reconocimiento de expresiones faciales emocionales a través de fotografías estáticas, los resultados indicaron que, al menos en el reconocimiento de las emociones básicas, los niños con TDAH en su conjunto no muestran un rendimiento menor (ni mayor) en el acierto de la expresión facial emocional. Sin embargo, nuestros resultados sí indican que estos niños muestran un mayor tiempo destinado a responder al estímulo, sobre todo en relación con las emociones desagradables de miedo, asco y enfado. Estos resultados concuerdan con el estudio de Guyer et al. (2007) en el que no se encontraron diferencias entre participantes con TDAH y participantes control en la ejecución de las pruebas relacionadas con el reconocimiento facial emocional. En dicha investigación se evaluó el nivel de ejecución en esta variable a través de fotografías estáticas en diferentes grupos: trastorno bipolar, trastornos del estado de ánimo, ansiedad y/o depresión mayor, TDAH y/o trastorno de conducta y un grupo control. Los resultados mostraron que las personas con trastorno bipolar y alteraciones en el estado de ánimo cometieron más errores que los sujetos con ansiedad, depresión, TDAH, trastorno de conducta y sujetos controles. De igual manera, otros estudios indican que los niños con TDAH y trastornos de conducta son tan precisos como los controles en el reconocimiento facial emocional (Cadesky, Mota, y Schachar, 2000). Sin embargo, los estudios de Corbett y Glidden (2000) y Pelc et al. (2006) contradicen los resultados obtenidos en nuestra investigación. En el primer trabajo se evaluó la capacidad de niños y niñas con TDAH de entre 7 y 12 años en la percepción de estímulos faciales emocionales y en la prosodia. Los resultados mostraron que los niños con TDAH presentaban déficits de nivel leve a moderado en la percepción de los estímulos emocionales.

En cuanto a los estímulos presentados de manera dinámica, en el presente estudio sí se encontraron diferencias en la ejecución de la prueba. El grupo TDAH obtuvo una menor precisión de respuesta y un tiempo de reacción mayor ante estos estímulos. Esto coincide con el estudio de Ludlow, Garrood, Lawrence, y Gutiérrez (2014) quienes compararon a un grupo clínico diagnosticado de TDAH y un grupo homólogo control en el grado de percepción del movimiento facial, tono de voz y gestos en las siete categorías emocionales básicas (miedo, ira, tristeza, asco, sorpresa, y alegría), así como en una categoría neutral. 
Una posible explicación a los diferentes resultados en función de si los estímulos se presentaban de forma estática o dinámica está relacionada con el hecho de que diversos estudios sugieren que las evaluaciones de las emociones en los niños con TDAH deben ser administradas mediante un método que sea más representativo de las experiencias diarias (McDonald, 2012; Yuill y Lyon, 2007). Los investigadores deberían de ser críticos debido a la poca validez ecológica de los estímulos presentados de forma estática, ya que existen algunas evidencias de que el juicio de la expresión facial está afectado por el movimiento (Richardson, Bowers, Bauer, Heilman, y Leonard, 2000). Sin embargo, las investigaciones realizadas en este ámbito no muestran un acuerdo total ya que difieren en la forma de evaluación y los estímulos presentados. No obstante, al igual que otras investigaciones al respecto (Yuill y Lyon, 2007), el presente estudio avala la existencia de dificultades en el reconocimiento de expresiones faciales en niños con TDAH, no solamente en su funcionalidad sino en la demora de respuesta. Estas conclusiones suponen implicaciones directas para el diagnóstico y para la intervención del TDAH, que actualmente se centra sobre todo en aspectos educativos cuando quizás debería dar más valor a la educación emocional para ayudar a este colectivo en las relaciones interpersonales.

Por último, al realizar el análisis discriminante, se identifican dos categorías de TDAH (TDAH como grupo clínico y TDAH como grupo control). El primer grupo, se ajusta al perfil de personas con una menor precisión de respuesta y tiempo de reacción más lento respecto al grupo control. En cambio, el segundo grupo de este análisis discriminante presenta un patrón totalmente opuesto, con un rendimiento si cabe superior no solo al grupo de TDAH citado, sino también al conjunto de niños del grupo control. Este subgrupo de TDAH tiene una precisión de respuesta mayor en el reconocimiento de las emociones de tristeza y miedo, y un tiempo de reacción menor que el grupo TDAH y el grupo control.

En suma, dentro del grupo TDAH encontramos dos subgrupos, uno de los cuales presenta un grado de sensibilización mayor a ciertas expresiones no verbales relacionadas con emociones desagradables. En cambio, el otro grupo, el más numeroso, no se diferencia con el grupo control en el grado de precisión de respuesta y sí en el tiempo de reacción, que es más lento. Esto viene a señalar la heterogeneidad del grupo clínico TDAH, y la necesidad de identificar en trastorno de TDAH no sólo en base a un criterio categórico ( $\mathrm{si} / \mathrm{no}$ ) sino como un continuo (grado de la sintomatología). De hecho, existen evidencias de que esta patología podría ser un continuo en el cual hay niños que no cumplen todos los criterios para el diagnóstico clínico de TDAH, pero que presenten algunos de los síntomas nucleares y que podrían tener comprometido el rendimiento en ciertas áreas. Se identifican factores clínicos, genéticos, ambientales y neurobiológicos asociados con el TDAH, o que lo relacionan con elevados niveles de síntomas de TDAH en la población general, que son insuficientes para validar el constructo diagnóstico del TDAH. Los «clásicos» TDAH son los diagnosticados según los criterios CIE o DSM. Estos serían los sujetos gravemente afectados por una enfermedad que presentaría formas menores con mucha frecuencia. Por tanto, los datos o conductas que se toman en consideración para valorar un TDAH deberían ser dimensionales, distribuyéndose en un continuo que va desde la normalidad hasta la patología (APA, 2014; Thongseiratch y Worachotekamjorn, 2016).

Es resumen, este estudio muestra que cuando se utiliza estímulos dinámicos, más ecológicos que los estáticos, y se hace uso de las claves contextuales y estímulos dinámicos, el colectivo de niños diagnosticados con TDAH es impreciso en la interpretación de las señales sutiles de la emoción, en contra de los resultados obtenidos en relación con los estímulos estáticos. Con ello, se pretende subrayar la importancia de proporcionar a los niños diagnosticados con TDAH mensajes explícitos y coherentes que complemente la expresión de la emoción, como, por ejemplo, un refuerzo verbal. En particular, la conciencia de etiquetado incorrecto de expresiones faciales emocionales como el enfado o la tristeza podría disminuir la intensidad de situaciones problemáticas que surgen de conductas disruptivas del niño y así mejorar su autoestima y su autoconcepto además de las relaciones interpersonales.

\section{Conflictos de intereses}

Los autores declaran que no existen conflictos de intereses.

\section{Referencias}

Adams, R., Flannery, K., Finn, P., Rizzo, A., \& Moes, E. (2009). Distractibility in attention deficit/hyperactivity disorder (ADHD). The virtual reality classroom. Child Neuropsychology, 15(2), 120-135.

Albert, J., López-Martín S., Fernández-Jaén A., y Carretil, L. (2008). Alteraciones emocionales en el trastorno por déficit de atención/hiperactividad: Datos existentes y cuestiones abiertas. Revista de Neurología, 47(1), 39-45.

American Psychiatric Association (2014). DSM-5. Diagnostic and statistical manual of mental disorders (5th Edition). Washington, DC, USA: Masson. 
American Psychiatric Association (1994). Diagnostic and Statistical Manual of Mental Disorders, 4th edition (DSM$I V)$. Washington, DC: Masson.

Anton, R., Opris, D., Dobrean, A., David, D., \& Rizzo, A. S. (2009). Virtual reality in the rehabilitation of attention deficit hyperactivity disorder. Instrument construction. Principles Journal of cognitive and Behavioral psychotherapies, 9(2), 235-246.

Brown, T.E. (2009). ADHD Comorbidities. Arlington, USA: American Psychiatric Publishing.

Cadesky, E.B., Mota, V.L., \& Schachar, R.J. (2000). Beyond words: How do problem children with ADHD and/or conduct problems process nonverbal information about affect? Journal of the American Academy of Child and Adolescent Psychiatry, 39(9), 1160-1167.

Corbett, B. \& Glidden, H. (2000). Processing affective stimuli in children with attention-deficit hyperactivity disorder. Child Neuropsychology, 6(2), 144-155.

Fairchild, G., Van Goozen, H.M., Calder, A.J., Stollery, S.J., \& Goodyer, I.M. (2009). Deficits in facial expression recognition in male adolescents with early-onset or adolescence onset conduct disorder. Journal of Clinical and Experimental Neuropsychology, 50(5), 627-636.

Fenstermacher, K., Olympia, D., \& Sheridan, S.M. (2006). Effectiveness of a computer-facilitated, interactive social skills training program for boys with attention deficit hyperactivity disorder. School Psychology Quarterly, 21(2), 197-224.

Fox, J., Arena, D., \& Bailenson, J.N. (2009). Virtual Reality a survival guide for the social scientist. Journal of Media Psychology, 21(3), 95-113.

Garza, M.S., Núñez, V.P.S., y Vladimirsky, G.A. (2007). Autoestima y locus de control en niños con trastorno por déficit de atención con hiperactividad. Boletín Médico del Hospital Infantil de México, 64(4), 231-237.

Gutiérrez-Maldonado, J., Alsina-Jurnet, I., Carvallo-Becío, C., Letosa-Porta, A., y Magallón-Neri, E. (2007). Aplicaciones clínicas de la realidad virtual en el ámbito escolar. Cuadernos de Medicina Psicosomática y Psiquiatría de Enlace, 82, 32-51.

Gutiérrez-Maldonado, J., Letosa-Porta, A., Rus-Calafell, M., \& Peñaloza-Salazar, C. (2009). The assessment of attention deficit hyperactivity disorder in children using continuous performance tasks in virtual environments. Anuario de Psicología, 40(2), 211-222.

Guyer, A.E., McClure, E.B., Adler, A.D., Brotman, M.A., Rich, B.A., Kimes, A.S., Pine, D.S., Ernst, M., \& Leibenluft, E. (2007). Specificity of facial expression labeling deficits in childhood psychopathology. Journal of Child Psychology and Psychiatry, 48(9), 863-871.

Kats-Gold, I., Besser, A., \& Priel, B. (2007). The role of simple emotion recognition skills among school aged boys at risk of ADHD. Journal of Abnormal Child Psychology, 35(3), 363378.

Kessler, H., Bayerl, P., Deighton, R.M., \& Traue, H.C. (2002). Facially expressed emotion labeling (FEEL): pc-gestützer test zur emotionserkennung. Verhaltenstherapie und Verhaltensmedizin, 23(3), 297-306.

Lázaro, E.; Amayra, I., López-Paz, J. F., Martínez, O., Oliva, M., Pérez, M., Jometón, A., Berrocoso, S., García, M., Parada, P., Al Rashaida, M., \& Hoffman, H. (2016). Instrument for assessing the ability to identify emotional facial expressions in Healthy children and in children with ADHD: The FEEL Test. Journal of Attention Disorders, 0(0), 1-14.

Ludlow, A.K., Garrood, A., Lawrence, K., \& Gutierrez, R. (2014). Emotion recognition from dynamic emotional displays in children with adHD. Journal of Social and Clinical Psychology, 33(5), 413-427.

McDonald, S. (2012). New Frontiers in neuropsychological assessment: assessing social perception using a standardised instrument, the awareness of social inference test. Australian Psychologist, 47(1), 39-48.

Nakhuntina, L., Borod, J., \& Zgaljardic, D. (2006). Posed prosodic emotional expression in unilateral stroke patients: Recovery, lesion location, and emotional perception. Archives of Clinical Neuropsychology, 21(1), 1-13.

Nixon, E. (2001). The social competence of children with attention deficit hyperactivity disorder: A review of the literature. Child psychology y Psychiatry, 6(4), 172-180.

Rapport, L.J., Friedman, S.R., Tzelepis, A., \& Van Voorhis, A. (2002). Experienced emotion and affect recognition in adult attention-deficit hyperactivity disorder. Neuropsychology, 16(3), 102-110.

Pelc, K., Kornreich, C., Foisy, M.L., \& Dan, B. (2006). Recognition of emotional facial expressions in attention-deficit hyperactivity disorder. Pediatric Neurology, 35(2), 93-97.

Polanczyk, G., Lima, M.S., Horta, B.L., Biederman, J., \& Rohde, L.A. (2007). The worldwide prevalence of ADHD: A systematic review and metaregression analysis. American Journal of Psychiatry, 164(6), 942-8.

Richardson, C.K., Bowers, D., Bauer, R.M., Heilman, K.M., \& Leonard, C.M. (2000). Digitizing the moving face during dynamic displays of emotion. Neuropsychologia, 38(7), 10281039.

Rizzo, A.A., Buckwalter, J.G., Bowerly, M.S., Van der Zaag, C., Humphrey, L., Neumann, U., Chua, C., Kyriakakis, C., Van Rooyen, A., \& Sisemore, D. (2004). The virtual classroom: A virtual reality environment for the assessment and rehabilitation of attention deficits. CyberPsychology $y$ Behaviour, 3(3), 483-499.

Rodríguez, F. (2010). El trastorno por déficit de atención con hiperactividad: causas e implicaciones para el tratamiento. Psicología Educativa, 16(1), 31-40.

Rubiales, J., Urquijo, S., Said, A., y Macbeth, G. (2017). Proceso de toma de decisiones en niños y adolescentes con TDAH: Revisión sistemática. Revista de Psicopatología y Psicología Clínica, 22(2), 139-155.

Spitzer, V.M. \& Ackerman, M.A. (2008). The visible human at the university of colorado 15 years later. Virtual Reality, 12(4), 191-200.

Sinzig, J., Morsch, D., \& Lehmkuhl, G. (2008). Do hyperactivity, impulsivity and inattention have an impact on the ability of facial affect recognition in children with autism and ADHD? European Child \& Adolescent Psychiatry, 17(2), 63-72.

Thongseiratch, T. \& Worachotekamjorn, J. (2016). Impact of the DSM-V Attention Deficit Hyperactivity Disorder Criteria for Diagnosing Children with High IQ. Psychological Reports, 119(2), 365-373.

Villanueva-Bonilla, C. y Ríos-Gallardo, A.M. (2018). Factores protectores y de riesgo del trastorno de conducta y del 
trastorno de déficit de atención e hiperactividad: Una revisión sistemática. Revista de Psicopatología y Psicología Clínica, 23(1), 59-74.

Williams, L.M., Hermens, D.F., Palmer, D., Kohn, M., Clarke, S., Keage, H., Clarck, C.R., \& Gordon, E. (2008). Misinterpreting emotional expressions in attention-deficit/hyperactivity disorder: Evidence for a neural marker and stimulant effects. Biological Psychiatry, 63(10), 917-926.

Yuill, N. \& Lyon, J. (2007). Selective difficulty in recognizing facial expressions of emotion in boys with ADHD. General performance impairments or specific problems in social cognition? European Child and Adolescent Psychiatry, 16(6), 398-404. 